\title{
AS RELAÇÕES ENTRE POBREZA E BEM-ESTAR: UMA REVISÃo SISTEMÁTICA
}

\author{
James Ferreira Moura Junior \\ Universidade da Integração Internacional da Lusofonia Afro-Brasileira \\ Jorge Castellá Sarriera \\ Universidade Federal do Rio Grande do Sul
}

\begin{abstract}
Resumo
O objetivo é analisar as relações entre pobreza e do bem-estar em artigos científicos. Os descritores da busca foram: pobreza, pobre, baixa renda e bem-estar. Foram selecionados os artigos produzidos entre 2010 até 2016. Os critérios de inclusão foram: ser uma pesquisa empírica com pessoas em situação de pobreza; utilizar a conceituação de bem-estar; ser um artigo completo; e ter adultos como amostra participante. Foram identificados 592 artigos, sendo analisadas 24 produções. O delineamento quantitativo é o mais utilizado junto com a perspectiva monetária de mensuração e conceituação da pobreza. O conceito de bem-estar subjetivo de Ed Diener é o mais presente. Identifica-se que a pobreza tem um impacto negativo no Bem-Estar Subjetivo, mas há paradoxos específicos que apontam tendências contrastantes. E importante apontar a necessidade de desenvolver investigações analisando o impacto da pobreza multidimensional no bem-estar.

Palavras-chave: Pobreza; Bem-Estar; Revisão Sistemática.
\end{abstract}

\section{THE RELATIONS BETWEEN POVERTY AND WELL-BEING: A SYSTEMATIC REVIEW}

\begin{abstract}
The aim is to analyze the relations between poverty and well-being in scientific papers. The search descriptors were poverty, poor, low income, and well-being. Articles produced from 2010 until 2016 were selected. The inclusion criteria were: be an empirical survey of people in poverty; use the well-being concept; be a complete article; and have adults as sample. We identified 592 articles, being examined 24 . The quantitative design is the most used with the monetary perspective of measurement and conceptualization of poverty. The concept of subjective well-being of Ed Diener is the most present. It identifies that poverty has a negative impact on the subjective well-being, but there are specific paradoxes that link contrasting trends. It is important to point out the necessity of developing investigations analyzing the impact of multidimensional poverty on well-being.
\end{abstract}

Keywords: Poverty; Well-Being; Systematic Review. 


\title{
RELACIONES ENTRE POBREZA Y BIENESTAR: UNA REVISIÓN SISTEMÁTICA
}

\begin{abstract}
Resumen
El objetivo es analizar las relaciones entre pobreza y bienestar en artículos científicos. Los descriptores de búsqueda fueron: pobreza, pobre, bajo ingreso y el bienestar. Fueron seleccionados los artículos producidos a partir de 2010 hasta 2016. Los criterios de inclusión fueron: ser un estudio empírico con personas en situación de pobreza; utilizar el concepto de bienestar; ser un artículo completo; y tener adultos como muestra. Fueron identificados 592 artículos, siendo examinadas 24 producciones. El diseño cuantitativo es el más utilizado junto con la perspectiva monetaria de medida y conceptualización de la pobreza. El concepto del bienestar subjetivo de Ed Diener es el más presente. Se identifica que la pobreza tiene un impacto negativo sobre el Bienestar Subjetivo, pero hay paradojas específicas que apuntan tendencias contrastantes. Es importante señalar la necesidad de desarrollar investigaciones analizando el impacto de la pobreza multidimensional en el bienestar.

Palabras clave: Pobreza; Bienestar; Revisión Sistemática.
\end{abstract}

\section{INTRODUÇÃO}

O bem-estar pode ser entendido como a capacidade de o indivíduo ter liberdade de escolha (Sen, 2000). Dessa maneira, está vinculado com um ideal de autonomia relacionado a fatores externos e internos. Assim, Sarriera (2015) concebe o bem-estar como atravessado por questões contextuais, sociais e culturais. O estabelecimento do bem-estar pode ser compreendido como o estabelecimento de um espaço de justiça social onde os seres humanos deveriam ser contemplados (Sen, 2013). Portanto, o bem-estar está intimamente atrelado a uma realidade social que, no Brasil, é atravessada por uma forte desigualdade social e pobreza (Moura Jr., Ximenes, \& Sarriera, 2014). Assim, tem-se como objetivo no presente estudo analisar as relações entre pobreza e do bem-estar em artigos científicos a partir da análise de artigos científicos nacionais e internacionais pulicados nos últimos anos".

Primeiramente, salienta-se que há diferentes formas de conceber o bemestar. Paloutzian e Ellison (1982) apresentam o conceito de bem-estar existencial vinculado a sentimentos de satisfação e propósito com a vida. Já Elliott e Aseltine (2013) trazem o conceito de bem-estar emocional que se vincula com um estado de não sofrimento. O bem-estar pessoal está mais vinculado a satisfação com âmbitos concretos da vida de forma central e aspectos periféricos relacionados à autoestima, percepção de controle, apoio social percebido, otimismo vital, entre outros (Casas, 2010).

O bem-estar subjetivo seria o conjunto das respostas do indivíduo sobre sua satisfação com a vida (Diener, 1984). Salienta-se que este conceito é um dos mais utilizados para avaliar aspectos subjetivos da qualidade de vida e a felicidade (Diener, 2012). Segundo Albuquerque e Tróccoli (2004), o bem-estar subjetivo constitui-se como uma avaliação individual sobre a vida de maneira global e dinâmica a partir da preponderância de afetos positivos. De acordo com Distante (2013), este conceito vincula-se uma perspectiva hedônica de bem- 
estar baseada na prevalência do prazer e da gratificação. Ele seria o conjunto das respostas do indivíduo sobre sua satisfação com a vida com uma vinculação maior a aspectos subjetivos (Diener, Suh, Lucas, \& Smith, 1999; Cummins et al., 2003), refletindo sobre seu estado geral de bem-estar (Ryan \& Deci, 2001).

Segundo Machado e Bandeira (2012), a perspectiva eudemônica do bemestar surgiu da crítica ao bem-estar subjetivo relacionada ao questionamento sobre a centralidade da felicidade e do prazer para a existência humana. Dessa maneira, o bem-estar psicológico está relacionado ao desejo de autorrealização, desenvolvimento pessoal e busca de sentido de vida por meio das potencialidades de cada indivíduo. Entende-se que essa perspectiva representaria um processo de atualização da própria identidade a partir da autonomia, autoaceitação, identificação de um propósito para a vida, domínio sobre o ambiente, relações positivas com os outros e crescimento pessoal.

Apesar dessa crítica vinculada ao desenvolvimento do conceito de bemestar psicológico, compreende-se que o bem-estar subjetivo foi um dos conceitos e uma das medidas mais utilizadas nos estudos sobre impacto da renda na satisfação global com a vida (Diener, 2012). Igualmente, pode ser considerado uma das dimensões perdidas na compreensão do impacto da pobreza na vida dos indivíduos (Samman, 2007), pois as pesquisas que trabalham com renda de forma geral não necessariamente focam as consequências de uma realidade de pobreza. No entanto, identifica-se que as estratégias de mensuração e de conceituação da pobreza podem gerar diferentes impactos nos resultados das pesquisas desenvolvidas (Feres \& Villatoro, 2013). Essa mesma lógica pode ser utilizada pela conceituação de bem-estar subjetivo (Diener, $\mathrm{Ng}$, Harter, \& Arora, 2010; Howell \& Howell, 2008).

Pontua-se que há explicações que apontam a necessidade de investigar a preponderância desses aspectos subjetivos na relação com a pobreza. De acordo com Wang, Shang e Xu (2011), há a Teoria Absoluta, que concebe o bem-estar subjetivo estritamente relacionado à renda. Já a Teoria Relativa incorpora o status econômico da região ao longo do tempo a partir da comparação social e das expectativas do indivíduo sobre a renda relativa como constituintes do bemestar subjetivo. Igualmente, há teorias que são baseadas em condições não materiais, não importando a renda real do indivíduo. Elas seriam a: Teoria Adaptativa e a Teoria da Expectativa. Esta última estaria baseada nas expectativas totais do indivíduo como parâmetro para seu bem-estar subjetivo. Já a Teoria Adaptativa concebe que o indivíduo tem um poder de adaptação a partir de sua habilidade emocional a responder eventos positivos e negativos.

Nesta mesma lógica, deve-se também compreender que as concepções de pobreza são diversas, podendo estar baseadas em perspectivas unidimensionais e multidimensionais em diferentes linhas da ciência econômica. As tendências unidimensionais geralmente selecionam somente um indicador para mensurar a pobreza, estando relacionadas à privação de renda. De acordo com Soares 
(2009), as linhas de pobreza indicam um determinado patamar pautado geralmente em um quantitativo de renda para atestar uma situação de pobreza caso o indivíduo esteja abaixo desse parâmetro, fundamentando uma forma de mensuração monetária. Já as perspectivas multidimensionais baseiam-se em diferentes dimensões e indicadores para identificar uma situação de pobreza. Como exemplo, há a abordagem das necessidades humanas básicas que compreende a pobreza como privação nas dimensões saúde, educação e rendimentos (Comim \& Bagolin, 2002). Outra abordagem existente é Abordagem das Capacitações que concebe a pobreza como um estado de privação de liberdade (Sen, 2000).

Dessa forma, esse tipo de compreensão multidimensional acerca da pobreza abarca diferentes dimensões que não estão representadas na lógica do mercado, mas que afetam o bem-estar do indivíduo (Salama \& Destremau, 2002). A partir da abordagem das capacitações que se insere nesse âmbito multidimensional, a situação de pobreza instala-se quando há a privação de capacitações básicas (Alkire, 2007). Assim, devem-se entender as capacitações como os estados de privação de indivíduos que estão para além de uma questão monetária. De forma ilustrativa, concebem-se esses estados, como estar nutrido de forma satisfatória, de estar saudável, de viver sem sentir vergonha pela sua situação social, ser feliz, entre outros (Sen, 1996). Este estudo parte da compreensão que as abordagens multidimensionais da pobreza representam melhores formas de compreensão e de mensuração desse fenômeno, porque abrangem a complexidade dessa situação para além de uma medida única de privação de renda.

No entanto, informa-se que a maioria dos estudos realizados utilizam somente medidas monetárias unidimensionais para mensurar a pobreza, apontando que a sua redução pode causar uma diminuição na média do bemestar subjetivo (Biswas-Diener \& Diener, 2006; Hochman \& Skopek, 2013; Wilkinson \& Pickett, 2006; Tiliouine, Cummins, \& Davern, 2006). Além disso, constata-se a existência de um maior efeito negativo da privação de renda no bem-estar subjetivo em regiões mais pobres (Diener, 1984; Diener \& BiswasDiener, 2002; Wilkinson \& Pickett, 2006). Identificaram-se baixos índices de felicidade em pessoas pobres participantes de uma pesquisa na Argélia (Tiliouine et al., 2006). Apesar disso, esses resultados não podem ser considerados uma regra, segundo Graham (2009), com seu "paradoxo dos camponeses felizes e dos milionários miseráveis" (paradox of happy peasants and miserable millionaires). Diener, Sandvik, Seidlitz e Diener (1993), Diener et al. (1999), Huebner (2004) e Casas (2011) apontam que somente um estado de extrema pobreza fornece o impacto significativo na redução das médias do bem-estar subjetivo.

Compreende-se que há grupos específicos que mesmo vivendo situações significativas de pobreza não apresentam médias de bem-estar subjetivo 
reduzidas, sendo evidenciado que pode haver fatores culturais e contextuais mediando essa relação. Concebe-se que a relação entre pobreza e renda não é linear, porque pode ser identificado que as pessoas com menos renda tenham maiores índices de satisfação (Teschl \& Comim, 2005). Tanto para Webb (2009), que realizou uma investigação com grupos tibetanos, como para Biswas-Diener, Vitters $\varnothing$ e Diener (2005), que pesquisaram os grupos étnicos Kenyan Maasai, Us Amish e Greenlandic Inughuit nos Estados Unidos, observou-se que os indivíduos desses grupamentos portavam índices satisfatórios de felicidade mesmo imersos em situação de privação extrema de renda e de necessidades básicas.

No entanto, o aumento da renda não segue diretamente essa mesma constatação, não necessariamente elevando os índices de bem-estar. Geralmente, o crescimento da renda nas nações mais ricas não tem o mesmo impacto no aumento do bem-estar subjetivo que em países com grande parcela da população em situação de pobreza (Easterlin, 1974). Ou seja, o acréscimo da renda não significaria uma elevação no nível de bem-estar subjetivo em amostras de países com baixas porcentagens de pessoas em situação de pobreza. Essa constatação refere-se ao Paradoxo de Easterlin ou Paradoxo da Felicidade. Há uma relação evidente entre situação econômica e bem-estar subjetivo até um determinando ponto (Easterlin, 2001). Com o aumento da renda, o poder preditivo do dinheiro passa ser menos eficaz na promoção do bem-estar subjetivo (Diener et al., 2010; Teschl \& Comim, 2005). Por conta dessa diversidade de apontamentos, tem-se um tópico específico para analisar as relações entre pobreza e bem-estar subjetivo presentes nas produções identificadas nesta revisão sistemática.

Essa instabilidade nos apontamentos sobre a relação entre pobreza e bemestar subjetivo em populações em situação de riqueza e de pobreza advém da variedade de fatores que podem afetar essa relação (Guardiola, GonzalezGomez, Garcia-Rubio, \& Lendechy-Grajales 2012). As pessoas em situação de pobreza podem utilizar mecanismos de acomodação a uma realidade adversa, selecionando preferências possíveis para serem desejadas e alcançadas. Sedimenta-se um processo de preferências adaptativas que podem aumentar o nível de satisfação dos indivíduos mais pobres (Teschl \& Comim, 2005). Diener et al. (1993) argumentam que a renda tem pouco poder explicativo na variação do bem-estar subjetivo. Dessa maneira, segundo os mesmos autores, entendese que diferenças culturais, aspirações baseadas em um baixo parâmetro de avaliação e adaptação à situação de privação podem ser alguns dos fatores para explicar altos níveis de bem-estar subjetivo em pessoas em situação de pobreza.

Igualmente, quando se tem condições socioeconômicas adequadas, mesmo se os níveis de renda aumentarem, os níveis de bem-estar subjetivo se tornam estáveis ou até tem um crescimento abaixo do esperado. Entende-se que um dos aspectos que influenciam essa relação seria que as preferências pessoais relacionadas aos bens materiais são influenciadas por aspectos contextuais e 
pelo estrato social em que o indivíduo está imerso, constituindo os parâmetros para as comparações sociais (Easterlin, 2001; Boes, Staub, \& Winkelmann, 2010). Assim, essa perspectiva de avaliar o bem-estar subjetivo por meio de comparações sociais torna-se mais evidente quando a forma de mensuração da renda está baseada na abordagem relativa da pobreza (Comim \& Bagolin, 2002; Luttmer, 2005; Clark, Frijters, \& Shields, 2008). É importante salientar também que, de acordo com Monteserrat, Casas e Moura jr. (2015), a maioria dos estudos sobre bem-estar com pessoas em situação de pobreza é desenvolvida com indivíduos adultos. Por isso, essa revisão sistemática de literatura tem como foco essa população. Além dessas questões mais complexas vinculadas à realidade social e à pobreza, há uma profusão de definições e de nomenclaturas para o bem-estar, como já explicitado anteriormente.

\section{MÉTODO}

Emprega-se a técnica de revisão sistemática, pois esta é reconhecida por sua robustez na avaliação e síntese da literatura trabalhada. No entanto, é importante ressaltar que a qualidade desta técnica é estritamente relacionada à sua estrutura metodológica (Zoltowski, Costa, Teixeira, \& Koller, 2014). Utilizando diretrizes fornecidas por Fernández-Ríos e Buela-Casal (2009) sobre revisão sistemática de artigos, deve-se apresentar de forma precisa as estratégias de busca e os procedimentos analíticos utilizados. Foram usados descritores abrangentes para identificação do maior número possível de artigos que poderiam estar relacionados com as temáticas pobreza e bem-estar. Dessa maneira, foram utilizados os seguintes descritores com operadores booleanos em inglês: "Poverty" OR "Poor" OR "Low income" AND "Well-Being" nas bases de dados: Web of Science, Scopus e Sage. Nas bases de dados SCIELO, Lilacs e Pepsic, foram utilizados os mesmos operadores traduzidos para o português ("Pobreza" OU "Pobre" OU "Baixa renda" E "Bem-Estar") e para o espanhol ("Bienestar" O "Pobre" O "Baja renta" $Y$ "Bienestar") e realizadas novas buscas.

Foram buscados artigos nas áreas das Ciências Humanas e Sociais. Foram selecionados os artigos produzidos entre 2010 até novembro de 2016. Um dos primeiros critérios de inclusão foi ser uma pesquisa empírica com pessoas em situação de pobreza, ou seja, mencionar a condição econômica de privação dos participantes da investigação. Também, são considerados como critérios de inclusão: apresentar menção que é uma pesquisa que utiliza o conceito de bemestar; ser um artigo completo; e ter adultos como amostra participante. 0 critério de exclusão foi a presença de investigações empíricas sobre os impactos de substâncias químicas, como medicamentos, no bem-estar dos indivíduos, porque estes estudos restringem o escopo de análise somente ao aspecto medicamentoso, não sendo o objetivo dessa produção. 
Dessa maneira, os procedimentos de busca com os resultados gerais estão presentes na Figura 1:

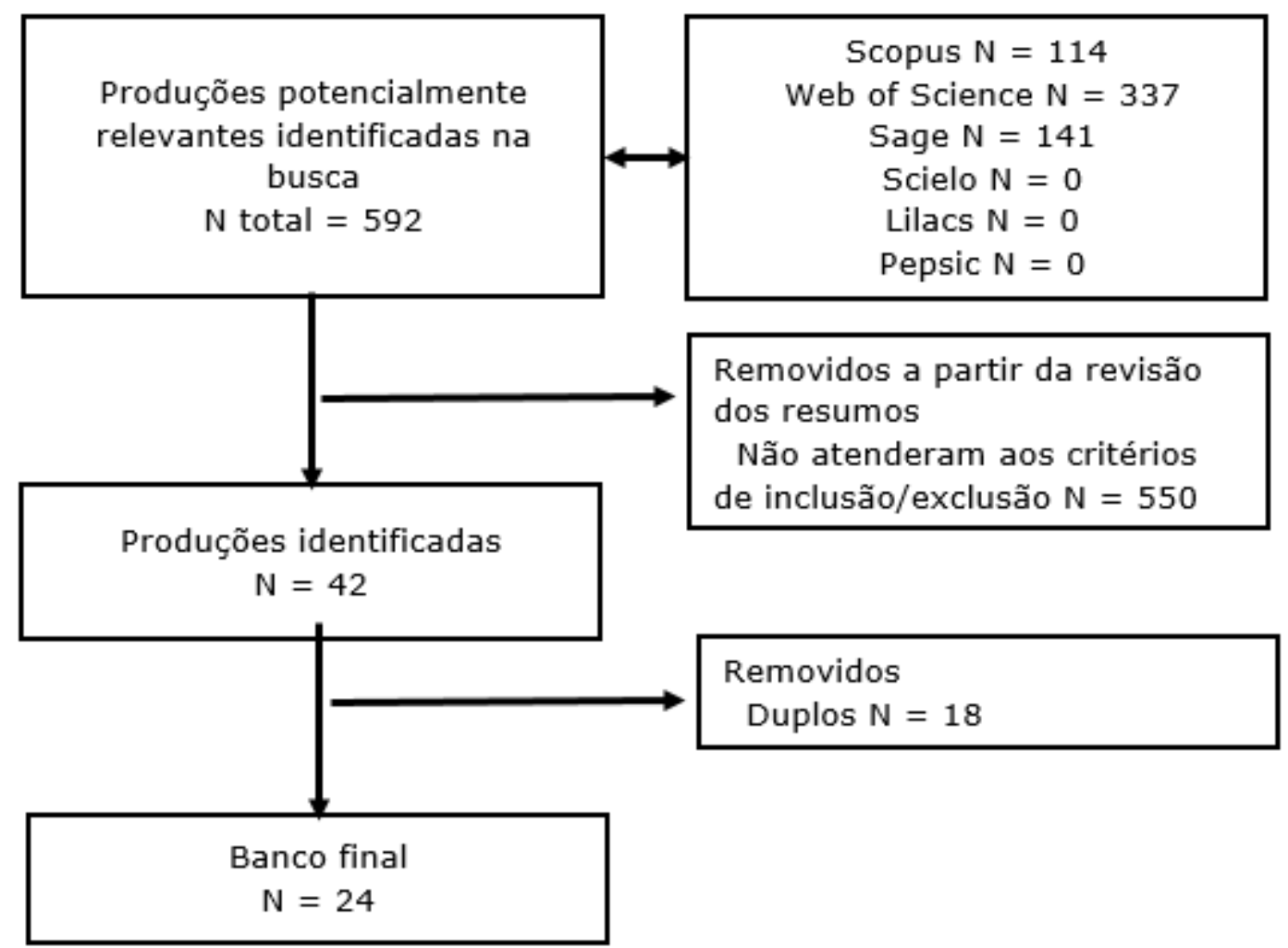

Figura 1. Procedimentos de busca e resultados gerais

É importante salientar que os critérios de filtro com uso dos descritores foram aplicados da mesma maneira nas bases pesquisadas. Nas bases de dados Scielo, Lilacs e Pepsic não foram identificadas produções que trabalharam com pobreza e bem-estar de forma conjunta. Assim, na Web of Science, foram identificados de forma geral 337 artigos. Aplicando-se os critérios de inclusão e de exclusão, restaram 21 artigos. Na Scopus, foram obtidos 114 artigos em um primeiro momento. Com os critérios, restaram 10 produções. Na base Sage, foram encontrados 141 artigos. Aplicando as normas de inclusão e de exclusão, foram selecionados 11 artigos. Assim, no total, foram obtidos 42 artigos para análise. Depois desse processo, foi realizada a análise novamente dos critérios e a identificação de artigos repetidos com a participação de um segundo juiz. Com isso, o número final de artigos para análise foi 24 . As categorias utilizadas para analisar as produções encontradas foram: base de dados pesquisada, periódico em que o artigo foi publicado, título do artigo, nome dos autores, ano de publicação, país de realização do estudo, conceito de bem-estar, conceito de pobreza, formas de mensuração da pobreza, formas de mensuração do bemestar e relações entre pobreza e bem-estar subjetivo. Este último tópico específico foi criado por conta da literatura já consolidada, de acordo com Diener (2012), sobre a relação entre o conceito de bem-estar subjetivo e pobreza. 
É importante ressaltar que, segundo Fernández-Ríos e Buela-Casal (2009), a estratégia analítica está baseada em uma perspectiva integrativa. Tem-se como foco de análise a qualidade das produções relacionadas à estrutura metodológica e ao grau de aprofundamento das temáticas abordadas, buscando similitudes e diferenças entre as produções (Zoltowski et al., 2014).

\section{RESULTADOS}

Neste tópico, apresentam-se as principais características dos artigos analisados. Primeiramente, apresenta-se a Tabela $1 \mathrm{com}$ as principais características dos artigos incluídos. Sobre os países investigados nas pesquisas, observa-se que os Estados Unidos, seguidos da África do Sul, foram onde mais estudos sobre pobreza e bem-estar foram realizados considerando os critérios de inclusão utilizados. No entanto, em um panorama geral identifica-se que a maioria das pesquisas foram desenvolvidas em nações periféricas, como Nicarágua, Etiópia, Bangladesh, Peru, Índia, Brasil, Colômbia e México. Também, ocorreram pesquisas transculturais envolvendo países da Europa, da América Latina e um estudo global com 90 países de todos os continentes. Acerca dos anos de publicação, são quatro artigos de 2010, três artigos de 2011, oito artigos de 2012, seis artigos de 2013, um de 2014, um de 2015 e um de 2016. É importante salientar também que todos os artigos estavam em língua inglesa, mesmo utilizando descritores em espanhol e português em bases de periódicos ibero-americanas. Em relação aos delineamentos das pesquisas, foram identificados 19 artigos com uma perspectiva quantitativa baseadas em levantamentos. Foram encontrados quatro artigos com âmbito qualitativo, sendo que três deles realizaram métodos participativos, como pesquisa ação participante. E somente um artigo baseou-se a perspectiva mista de pesquisa.

Observa-se que há uma diversidade de revistas que veicularam artigos com as temáticas pobreza e bem-estar subjetivo. Foram analisadas a partir de grandes temáticas de aglutinação. Elas podem ser divididas em três grupos: 1) as revistas com uma perspectiva mais ligada à saúde e áreas correlatas, como Journal of Health Psychology, Journal of Clinical Psychology, European Journal of Public Health, Health \& Social Care In The Community, Canadian Journal of Public Health, Advances In Life Course Research e American Journal of Orthopsychiatry; 2) revistas mais ligadas a políticas sociais e de desenvolvimento: Social Policy \& Administration, European Journal of Development Research, Development Policy Review, Research in Transportation Economics, Social Policy and Society, Research in Social Stratification And Mobility, Social Indicators Research (3) e International Journal of Social Welfare; 3) e revistas relacionadas à Ciência Psicológica em suas mais diversas áreas, como Journal Of Psychology And Theology, Journal of Family Issues, Social Psychology Quarterly e Journal of Economic Psychology (2). 
Tabela 1.

Lista de artigos identificados na revisão sistemática com informações sobre o primeiro autor, título, ano e periódico.

\begin{tabular}{|c|c|c|c|}
\hline Autores & Título Abreviado & Ano & Periódico \\
\hline Cramm, M. & $\begin{array}{c}\text { Improving Subjective Well- } \\
\text { being of the Poor }\end{array}$ & 2010 & $\begin{array}{l}\text { Journal Health } \\
\text { Psychology }\end{array}$ \\
\hline $\begin{array}{c}\text { Copestake, } \\
\text { J. }\end{array}$ & $\begin{array}{c}\text { Measuring multidimensional } \\
\text { aspiration gaps }\end{array}$ & 2010 & $\begin{array}{l}\text { Development Policy } \\
\text { Review }\end{array}$ \\
\hline Currie, G. & $\begin{array}{l}\text { Investigating links between } \\
\text { transport disadvantage, social }\end{array}$ & 2010 & $\begin{array}{l}\text { Research in } \\
\text { Transportation }\end{array}$ \\
\hline Smith, L. & $\begin{array}{c}\text { Psychological Interventions in } \\
\text { the Context of Poverty }\end{array}$ & 2010 & $\begin{array}{c}\text { American Journal of } \\
\text { Orthopsychiatry }\end{array}$ \\
\hline Wang, $\mathrm{X}$. & $\begin{array}{c}\text { Subjective Well-being Poverty } \\
\text { of the Elderly }\end{array}$ & 2011 & $\begin{array}{l}\text { Social Policy \& } \\
\text { Administration }\end{array}$ \\
\hline $\begin{array}{l}\text { Cruz-Ramos, } \\
\text { S. }\end{array}$ & Giving voice to the voiceless & 2011 & $\begin{array}{c}\text { Journal of Psychology } \\
\text { and Theology }\end{array}$ \\
\hline Rojas, M. & $\begin{array}{l}\text { Poverty and psychological } \\
\text { distress in Latin America }\end{array}$ & 2011 & $\begin{array}{l}\text { Journal of Economic } \\
\text { Psychology }\end{array}$ \\
\hline Perry, B. L. & Too Much of a Good Thing? & 2012 & $\begin{array}{c}\text { Social Psychology } \\
\text { Quarterly }\end{array}$ \\
\hline Martos, $\mathrm{T}$. & Life Goals and Well-Being & 2012 & $\begin{array}{l}\text { Social Indicators } \\
\text { Research }\end{array}$ \\
\hline $\begin{array}{c}\text { Cramm, J. } \\
\text { M. }\end{array}$ & $\begin{array}{l}\text { Individual- and } \\
\text { Neighbourhood-Level }\end{array}$ & 2012 & $\begin{array}{l}\text { Social Indicators } \\
\text { Research }\end{array}$ \\
\hline Fenge, L.A. & $\begin{array}{l}\text { The impact of the economic } \\
\text { recession on well-being }\end{array}$ & 2012 & $\begin{array}{c}\text { Health \& Social Care in } \\
\text { the Community }\end{array}$ \\
\hline $\begin{array}{l}\text { Asadullah, } \\
\text { M. N. }\end{array}$ & $\begin{array}{l}\text { Subjective well-being and } \\
\text { relative poverty in rural }\end{array}$ & 2012 & $\begin{array}{c}\text { Journal of Economic } \\
\text { Psychology }\end{array}$ \\
\hline Cox, K. & Happiness and Unhappiness & 2012 & $\begin{array}{c}\text { Journal of Happiness } \\
\text { Studies }\end{array}$ \\
\hline $\begin{array}{l}\text { Pedersen, } \\
\text { P.V. }\end{array}$ & $\begin{array}{c}\text { Associations between deprived } \\
\text { life }\end{array}$ & 2012 & $\begin{array}{l}\text { European Journal of } \\
\text { Public Health }\end{array}$ \\
\hline $\begin{array}{l}\text { Barrientos, } \\
\text { A. }\end{array}$ & $\begin{array}{c}\text { Poverty transitions among } \\
\text { older households }\end{array}$ & 2012 & $\begin{array}{l}\text { European Journal of } \\
\text { Development Research }\end{array}$ \\
\hline Gosh, S. & Citizenship in Practice & 2013 & $\begin{array}{l}\text { Journal of Asian and } \\
\text { African Studies }\end{array}$ \\
\hline Elliott, $\mathrm{S}$. & Being a Good Mom & 2013 & $\begin{array}{l}\text { Journal of Family } \\
\text { Issues }\end{array}$ \\
\hline Appio, L. & $\begin{array}{c}\text { Listening to the Voices of the } \\
\text { Poor and Disrupting }\end{array}$ & 2013 & $\begin{array}{l}\text { Journal of Clinical } \\
\text { Psychology }\end{array}$ \\
\hline Distante, $\mathrm{R}$. & $\begin{array}{c}\text { Subjective Well-Being, Income } \\
\text { and Relative }\end{array}$ & 2013 & $\begin{array}{l}\text { Social Indicators } \\
\text { Research }\end{array}$ \\
\hline Margolis, R. & Family, money, and health: & 2013 & $\begin{array}{l}\text { Advances In Life } \\
\text { Course Research }\end{array}$ \\
\hline Guardiola, J. & $\begin{array}{l}\text { Does higher income equal } \\
\text { higher levels of happiness }\end{array}$ & 2013 & $\begin{array}{c}\text { International Journal of } \\
\text { Social Welfare }\end{array}$ \\
\hline Oish, S. & $\begin{array}{l}\text { Residents of Poor Nations } \\
\text { Have a Greater Sense }\end{array}$ & 2014 & Psychological Science \\
\hline Prady, S. L. & $\begin{array}{l}\text { Does administrative data } \\
\text { reflect individual }\end{array}$ & 2015 & $\begin{array}{l}\text { Social Policy and } \\
\text { Society }\end{array}$ \\
\hline
\end{tabular}




Pitt, R. $\begin{gathered}\text { Low-income working } \\ \text { immigrant families }\end{gathered} \quad \begin{gathered}\text { Canadian Journal of } \\ \text { Public Health }\end{gathered}$

Nos tópicos seguintes, serão discutidos os conceitos e as formas de mensuração do conceito de bem-estar e pobreza utilizados nos artigos encontrados na revisão sistemática desenvolvida por esse estudo.

Conceitos e formas de mensuração do bem-estar

Os artigos foram agrupados a partir dos conceitos de bem-estar e suas estratégias de mensuração. Os conceitos de bem-estar são diversos, podendo ser identificados como existencial, emocional, subjetivo e psicológico. O conceito mais utilizado é o Bem-Estar Subjetivo de Diener (1984) junto com a Escala de Satisfação com a Vida (Satisfaction with Life Scale) (Diener, Emmons, Larsen, \& Griffin, 1985) e o item único da escala Overall Life Satisfaction (OLS) (Campbell, 1976). Depois desse instrumento, a outra escala utilizada, que é validada por outros estudos, é a Escala de Bem-Estar Existencial (Paloutzian, \& Ellison, 1982). E há também a Escala de Bem-estar Psicológico presente na pesquisa GallupHealthways Well-Being (Gasparini, Escudero, Marchionni, \& Olivieri, 2008). Retirando essas três escalas psicométricas e o item único OLS, os outros instrumentos foram criados pelos próprios estudos.

Iniciando esse processo analítico, Gosh (2013), Smith e Romero (2010) e Elliott et al. (2013) trazem o conceito de bem-estar emocional, não havendo qualquer explicação sobre esse conceito e sobre sua forma de mensuração. Já Perry, Pullen e Oser (2012) utilizam o conceito de bem-estar existencial, que significa sentimentos de satisfação e propósito com a vida. Sua forma de mensuração seria a partir da Escala de Bem-Estar Existencial de 12 itens relacionados a aspectos existenciais, a expectativas futuras e a propósitos da vida com um ranking de resposta de seis pontos de acordo e desacordo (Paloutzian \& Ellison, 1982). Appio, Chambers e Mao (2013) e Rojas (2011) trazem o conceito de bem-estar psicológico estando relacionado com a ausência de sofrimento ou de estresse psicológico. Ele é medido a partir das considerações de Gallup-Healthways Well-Being (Gasparini et al., 2008). Esse grupo propõe que os indivíduos respondam sim ou não se sentiram as seguintes emoções um dia antes de responder o questionário: enjoyment, anxiety, sadness, boredom, depression, anger, love, e physical pain (Prazer, ansiedade, tristeza, tédio, depressão, raiva, amor e dor física).

Pedersen, Grønbæk e Curtis (2012) trazem o conceito de bem-estar pessoal. No entanto, também não fazem explanações sobre esse construto. Quando o mensuram, utilizam os ítens dicotômicos (sim/não) para as seguintes afirmações: "Have felt very discouraged, depressed or unhappy the past 14 days" ("Tenho me sentido muito desencorajado, deprimido ou infeliz nos últimos 14 dias"), "Have felt often stressed in daily life" ("Tenho me sentido estressado 
diariamente"), "Have felt full of energy only a little of the time, or not at all, the past month" ("Tenho me sentido cheio de energia apenas por pouco tempo ou de nenhuma forma no mês passado"), "Have felt tired all the time, or most of the time, the past month" ("Tenho me sentido cansado o tempo todo, ou na maioria das vezes, no mês passado").

Prady et al. (2015) utilizam o conceito de bem-estar subjetivo financeiro, verificando por meio de um item único se a renda era adequada ao estilo de vida da família. Pitt, Sherman e Macdonald (2016) utilizam o conceito de bem-estar sociocultural baseando-se em uma perspectiva qualitativa a partir das privações causadoras de isolamento, aculturação, dificuldade de compreensão da cultura local. Cramm, Moller e Nieboer, (2010; 2012), Barriento e Mase (2012), Copestake e Camfield (2010), Margolis e Myrskylae (2013), Distante (2013), Wang et al. (2011), Guardiola et al. (2012) e Cox (2012) utilizam o conceito de bem-estar subjetivo de Diener (1984). Eles consideram que o bem-estar é um conjunto de avaliações que as pessoas fazem das suas vidas e das situações que ocorrem com elas. O Bem-Estar Subjetivo é considerado uma medida unidimensional hedônica livre de contexto (Casas, 2010).

No entanto, somente Copestake e Camfield (2010) compreendem que o bem-estar subjetivo é importante para a mudança social e para o fortalecimento dos indivíduos em um nível mais coletivista. Em outro artigo, o conceito de bemestar subjetivo tem uma base eudemônica, diferenciando-se das perspectivas hedônicas (Distante, 2013). É importante mencionar que este último autor vai contra as considerações consolidadas na literatura porque, de acordo com Blanco e Valera (2007), o bem-estar psicológico seria considerado eudemônico constituído de autoaceitação, autonomia, domínio sobre o entorno, relações positivas com os outros e crescimento pessoal. É consenso que o bem-estar subjetivo de Diener (1984) vincula-se à perspectiva hedônica (Casas, 2010; Diener et al., 1985; 1993; 2010).

Currie et al. (2010) e Distante (2013) utilizam para mensurar o bem-estar subjetivo o item único "How Satisfied are you with life overall?" ("Quanto você está satisfeito com sua vida em geral?)" de sete pontos de satisfação. Este item refere-se à escala Overall Life Satisfaction (OLS) (Satisfação Global com a Vida) (Campbell, 1976), mas é importante pontuar que os autores não a referenciaram em sua bibliografia. Margolis e Myrskylae (2013) também utilizam um item único, mas trabalham com uma afirmação diferente "All things considered, how satisfied are you with your life as a whole these days?" ("Considerando tudo, quanto você está satisfeito com a sua vida como um todo nos dias de hoje?") com uma escala likert de resposta de 10 pontos, partindo do insatisfeito ao satisfeito.

Martos e Kopp (2012) utilizam duas escalas desenvolvidas pelo próprio estudo para mensurar o bem-estar subjetivo: Self-rated Quality of Life e WHO Well-Being Index. Esta é uma escala de itens relacionada a ocorrência de estados 
de humor positivos nas últimas duas semanas, podendo ser respondido em um ranking de veracidade. Self-rated Quality of Life é uma escala de dois itens ("On the whole, how satisfied are you with your life" ("Em geral, quanto você está satisfeito com a sua vida") e "On the whole, how happy are you") ("Em geral, o quão feliz você está"). Já Barrientos e Mase (2012) não mensuram o bem-estar subjetivo.

De acordo com Cramm et al. (2010; 2012), como forma de mensuração, utilizam somente a escala Satisfaction with Life Scale (SWLS) que consiste em cinco itens sobre satisfação geral com a vida a partir de uma escala likert de 5 pontos de acordo e desacordo (Diener et al., 1985). Cox (2012) também utiliza o SWLS, mas expande a mensuração do bem-estar subjetivo também fazendo uso das escalas: Satisfaction with life domains (Satisfação com as diferentes dimensões da vida) e Memory for positive and negative events (Memória para eventos positivos e negativos). Este instrumento, que foi desenvolvido para a investigação realizada, funciona a partir do pedido de rememoração de eventos positivos e negativos, realizando um cálculo para identificação da prevalência desses tipos de eventos. E a outra escala expõe 12 âmbitos específicos (material resources, friendship, morality, intelligence, food, romantic, relationship, family, physical appearance, self, income, housing e social life) (Recursos materiais, amizade, moralidade, inteligência, alimentação, romance, relacionamento, família, aparência física, autoconceito, renda, habitação e vida social) avaliados por meio de um ranking de satisfação pelo indivíduo. Copestake e Camfield (2010) utilizam uma adaptação do Satisfaction with Life Scale (SWLS) e da Escala de Afetos Positivos e Negativos (PANAS) (Watson, Clark, \& Tellegen, 1988) para criar uma nova escala chamada de Escala de Escores de Qualidade Vida.

Wang et al. (2011) utilizam a Satisfaction with Life Scale (SWLS) para entender o bem-estar subjetivo como uma medida de pobreza. Deste modo, caso o participante tenha um baixo bem-estar, ele pode ser considerado pobre. Dessa maneira, o estudo desses autores baseia-se na perspectiva do bem-estar subjetivo ligado a condições não materiais. Como já foi criticada, a utilização de medidas de satisfação para mensurar a situação de pobreza é problemática por conta da influência de fatores adaptativos nesse processo de avaliação individual (Teschl \& Comim, 2005).

Guardiola et al. (2012) apresentam um item relacionado à felicidade de maneira geral "In general terms, how happy do you feel with your life?" ("Em termos gerais, o quão feliz você se sente com sua vida?"). E utilizam outra pergunta relacionada à felicidade, mas por âmbitos, com a questão "How happy do you feel in relation to... ?" ("Quão feliz você se sente em relação a..."). Em seguida, apresentam os âmbitos: health, work, love, money, quality of housing, nutrition, water, leisure, community of residence e trust of others (Saúde, trabalho, amor, dinheiro, qualidade da habitação, nutrição, água, lazer, 
comunidade de residência e confiança dos outros). Os participantes, então, responderam em uma escala likert de 0 a 10, com o menor sendo o mais baixo nível de felicidade e o maior o mais alto nível de felicidade. Apesar de Barriento e Mase (2012) apontarem considerações sobre esse conceito de bem-estar baseado nas considerações de Diener (1984), os autores não apresentam qualquer forma de mensuração desse construto.

Fenge et al. (2012) e Asadullah e Chaudhury (2012) utilizam o conceito de bem-estar subjetivo que também pode ser considerado como felicidade. No entanto, Fenge et al. (2012) o compreendem como constituído de bem-estar mental e bem-estar social. Este último seria o senso de pertencimento e participação em atividades sociais com família e amigos. Aquele seria vinculado à saúde mental e emocional, autoestima e aceitação das atuais condições da vida. Esses dois construtos estão em interação e integrariam a qualidade de vida do indivíduo. Como forma de mensuração, foram feitas perguntas abertas nas entrevistas qualitativas sobre o bem-estar mental e bem-estar social aos participantes. Asadullah e Chaudhury (2012) também elegem o item único "On the whole, how satisfied are you with your life?" ("Em geral, quão satisfeito você está com sua vida") respondido em uma escala likert de 10 pontos para mensurá-lo de acordo com a satisfação. Por fim, Currie et al. (2010) e Martos e Kopp (2012) apresentam somente o conceito de bem-estar geral, mas não fazem qualquer consideração teórica sobre ele, nem propõe estratégias de mensuração. No próximo tópico, avaliam-se os resultados apresentados nos artigos sobre as formas de conceituação e mensuração da pobreza.

\section{Conceitos e formas de mensuração da pobreza}

Foram identificados dois grandes grupos. No Grupo 1, com 10 artigos, estariam as produções sem nenhuma conceituação específica sobre pobreza, mas que poderiam ser compreendidas a partir da perspectiva monetária. Neste mesmo grupo, as formas de mensuração estavam estritamente baseadas na renda. No Grupo 2, com 14 artigos, surgem as conceituações baseadas nas abordagens multidimensionais com formas de mensuração mistas, utilizando perspectivas monetárias e multidimensionais. Assim, fazem parte do primeiro grupo de artigos as produções de Perry et al. (2012), Pitt et al. (2016), Pedersen et al. (2012), Margolis e Myrskylae (2013), Fenge et al., (2012), Martos e Kopp (2012), Elliott, Powell e Brenton (2013), Cox (2012) e Distante (2013).

Sobre as formas de mensuração da pobreza, Pitt et al. (2016), Perry et al. (2012) e Cox (2012) utilizam a renda familiar para configurar a situação de pobreza das participantes. Fenge et al. (2012) trabalham com a renda e o poder de compra. Distante (2013) também utiliza três formas de mensuração monetária, como a renda familiar, a renda relativa e a média de consumo dos moradores do bairro. Esta última é desenvolvida a partir do cálculo da renda relativa e da renda pessoal do indivíduo a partir da linha de pobreza de 
referência da localidade. Ainda no Grupo 1, Barrientos e Mase (2012) trazem explicitamente o conceito da pobreza vinculado a aspectos monetários relacionados à renda e ao poder de compra do indivíduo. Como forma de mensuração, utilizam a incidência da pobreza a partir da renda. Pedersen et al. (2012) usam o item único "Persons who often did not get enough food because they could not afford it" ("Pessoas que muitas vezes não recebiam comida suficiente porque não podiam pagar por ela"). Já Margolis e Myrskylae (2013) e Martos e Kopp (2012) mensuram a pobreza a partir da renda relativa a partir de itens únicos de comparação social.

No Grupo 2, Cramm et al. (2010; 2012), Rojas (2011), Guardiola et al. (2012) e Asadullah e Chaudhury (2012) indicam que o conceito de pobreza deve ser entendido como atrelado ao desenvolvimento humano por meio de uma perspectiva multidimensional. Cramm et al. (2012) mensuram a pobreza a partir do Townsend Index. Esse instrumento analisa a situação de privação do indivíduo, avaliando a ausência de recursos materiais, de posse de residência e de carro. Igualmente, esses últimos autores utilizam o índice Gini para mensurar a desigualdade social da região. Apesar desse pequeno avanço para uma perspectiva multidimensional, quando é necessário mensurar a pobreza de forma concreta, há o retorno para a compreensão monetária da pobreza. Assim, Cramm et al. (2010) mensuram a pobreza de forma monetária. Eles utilizam a renda familiar dividida em três níveis: baixa renda, renda média e alta renda. $\mathrm{E}$ Rojas (2011) também utiliza a renda familiar, porém estabelece o critério de renda familiar abaixo de $\$ 2.50$ dólares por dia para um indivíduo ser considerado pobre. Já Guardiola et al. (2012) trabalham somente a renda relativa do indivíduo. Asadullah e Chaudhury (2012) mensuram a pobreza de forma absoluta e relativa.

Outro autor inserido nessa tendência é Gosh (2013). Ele critica a perspectiva monetária da pobreza e amplia a compreensão da pobreza para uma questão de privação de liberdade a partir da estratégia de intervenção Self Help Groups, i.e., grupos de ajuda mútua desenvolvidos para dar suporte emocional junto com os moradores. No entanto, esse autor somente intitula essa compreensão multidimensional da pobreza em trechos introdutórios da sua produção, não aprofundando a temática. Como instrumento para mensurar a pobreza, ele somente aponta que a comunidade na Índia onde é realizada a pesquisa é considerada uma cidade em situação de pobreza.

Prady et al. (2015) utiliza o conceito de privação material a partir de uma série de itens que envolvem diferentes estados de privação, como ter férias por pelo menos uma semana no ano, possuir sapatos para inverno, sair com amigos ou família pelo menos uma vez por mês, ter dinheiro suficiente para decoração da casa, possuir poupança e realizar alguma atividade de lazer. É importante mencionar que a perspectiva multidimensional deve levar em conta aspectos 
contextuais (Comim \& Bagolin, 2002), como apontado por esses autores neste estudo no Reino Unido.

Wang et al. (2011) utilizam considerações da Abordagem das Capacitações, mas acrescentam a importância de parâmetros objetivos. Igualmente, eles também apontam que a pobreza deve ser entendida por meio do bem-estar subjetivo, criando o bem-estar subjetivo da pobreza. Identifica-se que eles utilizam Overall Life Satisfaction (OLS) (Campbell, 1976). Essa escala, então, funciona como uma medida de pobreza subjetiva, porque, segundo os autores, se o participante tem um baixo nível de satisfação ele é considerado pobre. Se tiver um alto índice de satisfação, é considerado em situação de não pobreza.

De forma semelhante, Copestake e Camfield (2010) informam que o conceito de pobreza subjetiva se baseia na forma como as pessoas pensam e sentem em um determinado contexto social. Assim, os autores realizaram uma pesquisa em duas fases. Na primeira fase de caráter qualitativo, foram conduzidas entrevistas para averiguar o que seria necessário para os indivíduos terem uma vida feliz. Com os resultados, foi construído um questionário com itens específicos de cada país participante (Peru, Bangladesh, Etiópia, Tailândia). Cada item, como saúde, educação, trabalho, amigos, comida, lazer, meditação, entre outros, deveria ser respondido primeiramente como necessário e, em segundo, lugar como realizado. Os autores, então, utilizam parâmetros subjetivos para indicar a situação de pobreza, mas relacionam também com uma compreensão concreta e operacional de mensuração da pobreza a partir dos critérios de realização.

Currie et al. (2010) tratam o conceito de pobreza de transporte, que significa a onerosidade do gasto com transporte público ou com seu próprio carro para se locomover na cidade. Os autores mencionam que a pobreza de transporte está constituída pela pobreza de tempo (time poverty). Esta se baseia no tempo levado para o indivíduo locomover-se na cidade. Essa pobreza de tempo é mensurada a partir da renda, do desemprego e da exclusão social devido aos gastos com transporte pessoal ou a falta de transporte público para locomoção.

Appio et al. (2013) e Smith e Romero (2010) compreendem a pobreza como prejudicial ao desenvolvimento humano, estando relacionada à exposição a eventos traumáticos, à insegurança alimentar e à estigmatização como relacionadas à pobreza. Smith e Romero (2010) mensuram a pobreza, mas não trazem nenhum critério bem definido, mencionando somente que trabalham com usuários de serviços psicoterápicos em situação de pobreza. Eles podem ser considerados os mais críticos dos presentes nessa revisão sistemática. Compreendem que a pobreza é consequência de uma sociedade desigual e opressora, percebendo-a como uma estratégia de manutenção do status quo a partir de práticas discriminatórias. Esses autores fundamentam sua perspectiva 
de compreensão da pobreza a partir do paradigma da libertação. Entretanto, a mensuração da pobreza de forma vaga também ocorre com Smith e Romero (2010), porque somente mencionam que trabalham em uma pesquisa ação participante em uma comunidade economicamente pobre e marginalizada dos Estados Unidos. No próximo tópico, passa-se a analisar as relações entre bemestar subjetivo e pobreza de forma específica.

Relações entre bem-estar subjetivo e pobreza

É importante ressaltar que os artigos analisados neste tópico foram somente aqueles que utilizaram o conceito específico de bem-estar subjetivo em suas considerações, porque, dessa maneira, é possível comparar com a literatura já consolidada. Assim, Cramm et al. (2010) observaram que o grupo de pessoas com renda mais baixa tinha um nível de bem-estar subjetivo com a menor média em comparação com o grupo com renda intermediária e alta na África do Sul. Asadullah e Chaudhury (2012) realizaram uma pesquisa com 2318 pessoas em situação de pobreza e não pobreza de áreas rurais de Bangladesh, verificando que os indivíduos mais pobres têm índices mais baixos de satisfação comparado com as pessoas mais ricas das comunidades pesquisadas. Cox (2012) verificou que o grupo com menos pobreza difere significativamente de todos os outros, tendo média mais elevada de bem-estar subjetivo na Nicarágua.

Cramm et al. (2012) verificaram que o índice de privação multidimensional tem uma correlação negativa e significativa com o bem-estar subjetivo em Western Cape na África do Sul. Currie et al. (2010) também identificaram que a exclusão social funciona como uma variável preditora negativa do bem-estar subjetivo na Austrália. Utilizando somente análises descritivas, Distante (2013) observa que o aumento da renda tem um impacto positivo no bem-estar subjetivo em um recorte transversal no Reino Unido. No entanto, quando se avalia o impacto da renda a partir de uma perspectiva temporal, não há uma mudança significativa do bem-estar subjetivo com o aumento de renda. Observa-se, assim, que há a corroboração do Paradoxo de Easterlin (Easterlin, 2001) em que a renda não funciona como um parâmetro significativo de impacto no bem-estar subjetivo.

Seguindo com contribuições nessa mesma perspectiva do paradoxo, Margolis e Myrskylae (2013) observaram que a renda é mais importante para explicar a variância do bem-estar subjetivo em regiões pobres do que em regiões com mais riqueza. Estas últimas são consideradas Europa Ocidental e países anglo-saxões. Já as regiões mais pobres são África, Ásia, América Latina. Novamente por meio de uma questão temporal, identifica-se que nos países Anglo-saxônicos, na América Latina e na Europa Ocidental a variação da bemestar subjetivo pela renda é muito pequena, estando em torno de $1 \%$. Já o bemestar subjetivo tem uma variância explicada pela renda entre 5\% a 8\% na Ásia e África. Guardiola et al. (2012) verificaram, na população pesquisada no México, 
que, mesmo em condições de extrema pobreza, ela tem uma média de bemestar subjetivo elevada, sendo considerada mais alta do que as médias gerais de alguns países latino americanos, como Argentina, Colômbia, Costa Rica Nicarágua, Chile e México, legitimando o "Paradoxo dos camponeses felizes e dos milionários miseráveis".

\section{DISCUSSÃO}

Primeiramente, é importante evidenciar que as pesquisas em sua maioria foram desenvolvidas em países em situação de pobreza extrema e moderada e com forte desigualdade social, fornecendo possível validade ecológica para as investigações realizadas. Identifica-se que os artigos tinham como amostra participantes de países como Peru, Nicarágua, Índia, Etiópia, Tailândia, África do Sul, Brasil e Bangladesh, considerados com índices significativos de pobreza multidimensional de acordo com Índice Global de Pobreza Multidimensional do Oxford Poverty and Human Development Initiative (2016). Além disso, deve-se apontar sobre a diversidade de temáticas dos periódicos que serviram de veiculação aos artigos. No entanto, identifica-se que o bem-estar está mais visibilizado como uma categoria psicológica, pois há diversas revistas vinculadas à Ciência Psicológica. Verifica-se que há predominância da área da saúde. Igualmente, como a pobreza é uma temática abordada tanto na economia, como nas políticas sociais e de desenvolvimento humano, encontra-se igual presença de veículos de divulgação científica nesses âmbitos de conhecimento.

Apesar da preponderância de pesquisas quantitativas, é interessante observar que os métodos qualitativos são fundamentados a partir de perspectivas participativas, porque o trabalho com pessoas em situação de pobreza pode estar pautado em um compromisso do pesquisador com a transformação da realidade amparada pela participação social (Montero, 2006). Em relação aos delineamentos de pesquisa utilizados, avalia-se que há predominância das pesquisas quantitativas por conta das temáticas trabalhadas que são usualmente mensuradas a partir de escalas psicométricas e de instrumentos de identificação da intensidade e da abrangência da pobreza.

Seguindo essa perspectiva, nota-se a preponderância de estudos baseados na perspectiva unidimensional e monetária da pobreza, pois a maioria das pesquisas utiliza somente a privação de renda para mensurar a pobreza. Há uma problemática evidente com o uso dessa perspectiva, porque pode haver uma subestimação do número de pessoas em situação de pobreza quando se utiliza somente o indicador deficiência de renda (Feres \& Villatoro, 2013). De acordo com Lelli (2001), a pobreza é um fenômeno de difícil delimitação e mensuração, pois é considerada uma situação complexa que precisa de múltiplos indicadores para ser analisada. No entanto, quando há a utilização de vertentes mais críticas e multidimensionais, diminui-se a dificuldade de identificação da pobreza em sua 
complexidade de forma parcial, pois se aponta que as formas de mensuração utilizadas geralmente reproduzem as estratégias de mensuração unidimensionais e monetárias. Isso ocorre, porque geralmente as políticas públicas e as formas de mensuração da pobreza vigentes - que não são consensuais - somente restringem suas análises e suas ações para os indivíduos empobrecidos a partir de sua renda (Soares, 2009). Há, então, a incompreensão do fenômeno da pobreza em sua totalidade, menosprezando os aspectos sociais, estruturais e psicológicos constituintes desse fenômeno (Alkire, 2007; Sen, 2000).

Observa-se que as pesquisas que utilizam fundamentações multidimensionais e não se estruturam em formas de mensuração monetária utilizam estratégias de mediação da pobreza amparadas por aspectos subjetivos. Ou seja, utilizam parâmetros não concretos para identificar estados de privação. Comim (2005) comenta que essa abordagem pode dificultar a identificação factual da situação de pobreza. Há uma série de críticas sobre essas formas de mensuração multidimensional da pobreza baseadas somente em aspectos subjetivos para informar sua situação de pobreza. Aponta-se que critérios unicamente subjetivos para avaliar a pobreza são demasiadamente cambiáveis e individuais para criação de um parâmetro demonstrativo da situação de pobreza, porque há a influência das preferências adaptativas que podem aumentar a satisfação do indivíduo mesmo em uma situação de pobreza extrema. Essas preferências são mecanismos psicológicos de adaptação à realidade adversa, diminuindo os sentimentos de frustração e incômodo a partir da reestruturação não consciente das necessidades e expectativas do indivíduo (Teschl, \& Comim, 2005).

Sobre o conceito de bem-estar, é importante ressaltar que muitas produções não apresentavam ou indicavam os instrumentos que utilizavam para mensurá-lo. Alguns deles, quando indicavam a estratégia de mensuração, não a referenciava na bibliografia, impossibilitando a análise. Assim, identifica-se que há uma profusão de diferentes conceitos e formas de mensuração do bem-estar, como bem-estar pessoal, psicológico, existencial, emocional e subjetivo. Avaliase como negativa essa falta de consenso, porque se impossibilita a comparação validada dos diferentes resultados de investigação a partir de diferentes concepções teóricas e medidas psicométricas. Também foi observado que há diversos estudos que criam medidas psicométricas específicas, como também utilizam somente itens únicos para mensurar o bem-estar. Segundo Diener et al. (1999), historicamente as medidas utilizadas para mensurar este construto são diversas, sem a existência de consenso estabelecido sobre a melhor forma de mensurar o bem-estar. De acordo com Spaan (2007), não é adequado a utilização de medidas psicométricas somente com um único item, deve-se utilizar diferentes itens com equilíbrio na utilização de indicadores objetivos e subjetivos. Torna-se, dessa maneira, a medida mais confiável e fidedigna, propiciando mais robustez a escala psicométrica. Verifica-se que o conceito Bem-Estar Subjetivo 
de Diener (1985) alcançava essas premissas quando estratégia de mensuração estava baseada na Escala de Satisfação com a vida (Diener et al., 1985), sendo a escala mais utilizada nos artigos que continham estratégias de medidas do conceito.

Também, foram avaliadas as relações entre esse conceito de bem-estar subjetivo e pobreza, concebe-se que a maioria das análises realizadas tem como resultados impactos negativos nas médias de bem-estar subjetivo em virtude da pobreza tanto na perspectiva multidimensional, como na unidimensional monetária. No entanto, foi identificado que Distante (2013) e Margolis e Myrskylae (2013) seguem os resultados do paradoxo de Easterlin. Quando se tem condições socioeconômicas adequadas, os níveis de renda podem aumentar, mas os níveis de bem-estar subjetivo se tornam estáveis ou até tem um crescimento abaixo do esperado. Entende-se que um dos aspectos que influenciam essa relação seria que as preferências pessoais relacionadas aos bens materiais são influenciadas por aspectos contextuais e pelo estrato social em que o indivíduo está imerso, constituindo os parâmetros para as comparações sociais (Easterlin, 2001; Boes et al., 2010). Assim, essa perspectiva de avaliar o bem-estar subjetivo a partir de comparações sociais torna-se mais evidente quando a forma de mensuração da renda está baseada na abordagem relativa da pobreza (Comim \& Bagolin, 2002; Luttmer, 2005; Clark et al., 2008).

De forma contrastante, os resultados de Guardiola et al. (2012) estão de acordo com o paradoxo dos "camponeses felizes e dos milionários miseráveis" de Graham (2009). Esses resultados podem estar atrelados a esse último paradoxo, porque provavelmente a etnia Maya mesmo vivendo em condições de pobreza significativas não teria o hábito de utilizar a comparação social como balizador de sua satisfação. Diener et al. (1993) denotam que diferenças culturais, aspirações baseadas em um baixo parâmetro de avaliação e adaptação à situação de privação podem ser alguns dos fatores para explicar altos níveis de bem-estar subjetivo em pessoas em situação de pobreza. Assim, aponta-se a necessidade de seguir avançando nas pesquisas com a proposta de relacionar pobreza e bemestar, pois os resultados ainda não são consensuais, incidindo diversos fatores que ainda precisam ser analisados.

\section{CONSIDERAÇÕES FINAIS}

É identificado que há uma abrangência das investigações sobre a pobreza somente por meio de delineamentos de pesquisa quantitativistas. Há, assim, o detrimento da compreensão crítica e multidimensional da pobreza que poderia auxiliar na conceituação desse fenômeno de forma mais abrangente e detalhada. Sobre o bem-estar, há uma profusão de conceitos. A maioria deles não é perpassada por um embasamento teórico coerente e bem fundamentando. 
Igualmente, muitos utilizam somente itens únicos para mensurá-lo, podendo tornar a relação entre pobreza e esta categoria psicológica menos robusta estatisticamente.

Assim, há a necessidade de realização de mais estudos com base na perspectiva multidimensional da pobreza e com a utilização de escalas de bemestar mais robustas. Foi observado que os paradoxos desenvolvidos para explicar as relações entre bem-estar subjetivo e pobreza também estão presentes nos resultados da revisão sistemática. Por conta disso, é premente a realização de investigações transculturais para identificar fatores contextuais que incidem sobre a relações entre bem-estar subjetivo e pobreza. Além disso, observa-se a necessidade de realização de mais estudos qualitativos com as pessoas em situação de pobreza, pois, assim, abre-se um espaço para conhecimento e amplificação das vozes acerca dessa realidade de privação.

\section{REFERÊNCIAS}

Albuquerque, A. S., \& Tróccoli, B. T. (2004). Desenvolvimento de uma escala de bem-estar subjetivo. Psicologia: Teoria e Pesquisa, 20(2), 153-164.

Alkire, S. (2007). The missing dimensions of poverty data: An introduction. Oxford Poverty \& Human Development Initiative [OPHI]. Recuperado de http://www.ophi.org.uk/working-paper-number-0

Appio, L., Chambers, D.A., \& Mao, S. (2013). Listening to the voices of the poor and disrupting the silence about class issues in psychotherapy. Journal of Clinical Psychology, 69(2), 152-161. doi:10.1002/jclp.21954

Asadullah, M. N., \& Chaudhury, N. (2012). Subjective well-being and relative poverty in rural Bangladesh. Journal of Economic Psychology, 33, 940-950. doi: 10.1016/j.joep.2012.05.003

Barrientos, A., \& Mase, J. (2012). Poverty transitions among older households in Brazil and South Africa. European Journal of Development Research, 24(4), 570-588. doi:10.1057/ejdr.2012.13

Biswas-Diener, R., \& Diener, E. (2006). The subjective wellbeing of the homeless and lessons for happiness. Social Indicators Research, 76, 185-205. doi:10.1007/s11205-005-8671-9

Biswas-Diener, R., Vittersø, J., \& Diener, E. (2005). Most people are pretty happy, but there is cultural variation: The Inughuit, the Amish, and the Maasai. Journal of Happiness Studies, 6, 205-226.

Blanco, A., \& Valera, S. (2007). Los fundamentos de la intervención psicosocial. In B. Amalio, \& J. Rodríguez. Intervención Psicosocial (pp. 134-156). Madrid: MacGrawHill.

Boes, S., Staub, K., \& Winkelmann, R. (2010). Relative status and satisfaction. Economics Letters, 109, 168-170. doi:10.1016/j.econlet.2010.08.027 
Campbell, A. (1976). Subjective measures of well-being. American Psychologist, 31, 177-223. doi:10.1037/0003-066X.31.2.117

Casas, F. (2010). El bienestar personal: Su investigación en la infancia y la adolescencia. Encuentros en Psicología Social, 5(1), 85-101.

Casas, F. (2011). Social indicators and child and adolescent well-being. Child Indicators Research, 4, 555-575. doi:10.1007/s12187-010-9093-z

Clark, A., Frijters, P., \& Shields, M. (2008). Relative income, happiness, and utility: An explanation for the Easterlin paradox and other puzzles. Journal of Economic Literature, 46(1), 95-144. doi:10.1257/jel.46.1.95

Comim, F. (2005). Capabilities and Happiness: Potential Synergies. Review of Social Economy, 63(2), 161-176. doi:10.1080/00346760500129871

Comim, F. V., \& Bagolin, I. P. (2002). Aspectos qualitativos da pobreza no Rio Grande do Sul. Ensaios FEE, 23, 467-490.

Cramm, J. M., Moller, V., \& Nieboer, A. P. (2010). Improving subjective wellbeing of the poor in the Eastern Cape. Journal Health Psychology, 15(7) 1012-1019. doi:10.1177/1359105310367833

Cramm, J. M., Moller, V., \& Nieboer, A. P. (2012). Individual and neighborhoodlevel indicators of subjective well-being in a small and poor eastern cape township: The effect of health, social capital, marital status, and income. Social Indicators Research, 105, 581-593. doi:10.1007/s11205-011-9790-0

Copestake, J., \& Camfield, L. (2010). Measuring multidimensional aspiration gaps: A means to understanding cultural aspects of poverty. Development Policy Review, 28(5), 617-633. doi:10.1111/j.1467-7679.2010.00501.x

Cox, K. (2012). Happiness and unhappiness in the developing world: Life satisfaction among sex workers, dump-dwellers, urban poor, and rural peasants in Nicaragua. Journal of the Happiness Studies, 13, 103-128.

Cummins, R. A., Eckersley, R., Pallant, J., Van Vugt, J., \& Misajon, R. (2003). Developing a national index of subjective well-being: The Australian Unity Wellbeing Index. Social Indicators Research, 64, 159- 190. Recuperado de http://www.deakin.edu.au/research/acqol/instruments/wellbeingindex/publi cations.php

Currie, G., Richardson, T., Smyth, P., Vella-Brodick, D., Hine, J., Lucas, K., ..., \& Stanley, J. (2010). Investigating links between transport disadvantage, social exclusion and well-being in Melbourne and updated results. Research in Transportation Economics, 29, 287-295. doi: $10.1016 /$ j.retrec.2010.07.036

Diener, E. (1984). Subjective well-being. Psychological Bulletin, 95, 542-575. doi:10.1037/0033-2909.95.3.542

Diener, E. (2012). New findings and future directions for subjective well-being research. American Psychologist, 67(8), 590-597. doi:10.1037/a0029541. 
Diener, E., \& Biswas-Diener, R. (2002). Will money increase subjective wellbeing? Social Indicators Research, 57, 119-169. doi:10.1023/A:1014411319119

Diener, E., Emmons, R, Larsen, J., \& Griffin, S. (1985). The satisfaction with life scale. Journal of Personality Assessment, 49(1), 71-75. doi:10.1207/s15327752jpa4901_13

Diener, E., Ng, W., Harter, J., \& Arora, R. (2010). Wealth and happiness across the world: Material prosperity predicts life evaluations, while psychosocial prosperity predicts positive feeling. Journal of Personality and Social Psychology, 99(1), 52-61. doi:10.1037/a0018066

Diener, E., Sandvik, E., Seidlitz, L., \& Diener, M. (1993). The relationship between income and subjective wellbeing: Relative or absolute? Social Indicators Research, 28, 195-223. doi:10.1007/BF01079018

Diener, E., Suh, E., Lucas, R., \& Smith, H. (1999). Subjective well-being: Three decades of progress. Psychological Bulletin, 125(2), 276-302. doi: 10.1037/0033-2909.125.2.276

Distante, R. (2013). Subjective well-being, income, and relative concerns in the UK. Social Indicators Research, 22(1), 35-44. doi:10.1007/s11205-0120083-Z

Easterlin R. A. (1974). Does economic growth improve the human lot? Some empirical evidence. In P. A. David \& M. W. Reder (Eds.), Nations and households in economic growth: Essays in honor of Moses Abramowitz (pp. 89-125). New York and London: Academic Press.

Easterlin, R. A. (1995). Will raising the incomes of all increase the happiness of all? Journal of Economic Behavior and Organization, 27, 35-47. doi:10.1016/0167-2681(95)00003-B

Easterlin, R. A. (2001). Income and happiness: Towards a unified theory. The Economic Journal, 111, 465-484. doi:10.1111/1468-0297.00646

Elliott, S., Powell, R., \& Brenton, J. (2013). Being a good mom: Low-income, black single mothers negotiate intensive mothering. Journal of Family Issues, 20(10), 1-20.

Elliott, S., \& Aseltine, E. (2013). Raising teenagers in hostile environments: How race, class, and gender matter for mothers' protective carework. Journal of Family Issues, 34, 719-744. doi:10.1177/0192513X12452253

Fernández-Ríos, L., \& Buela-Casal, G. (2009). Standards for the preparation and writing of psychology review articles. International Journal of Clinical and Health Psychology, 9(2), 329-344. doi:10.1177/0192513X13490279

Fenge, L.A., Hean, S., Worswick, L., Wilkinson, C., Fearnley, S., \& Ersser, S. (2012). The impact of the economic recession on well-being and quality of life of older people. Health and Social Care in the Community, 20(6), 617624. 
Feres, J. C., \& Villatoro, P. (2013). A viabilidade de se erradicar a pobreza: Uma análise conceitual e metodológica. Cadernos de Estudos Desenvolvimento Social em Debate, 15, 10-87.

Galinha, I., \& Ribeiro, J. L. P. (2005). História e evolução do conceito de bemestar subjetivo. Psicologia, Saúde e Doenças, 6(2), 203-214.

Gasparini, L., Escudero, W. S., Marchionni, M., \& Olivieri, S. (2008). Income, deprivation, and perceptions in Latin America and the Caribbean: New evidence from the Gallup World Poll. CEDLAS, BA. Recuperado de https://publications.iadb.org/bitstream/handle/11319/3248/Events\%20man agement\%20system\%3a\%20test\%20document.pdf?sequence=1

Graham, C. (2009). Happiness around the world: The paradox of happy peasants and miserable millionaires. Oxford: Oxford University Press.

Gosh, S. (2013). Citizenship in practice: Poverty reduction and self-help groups. Journal of Asian and African Studies, 1, 0-15. doi:10.1177/0021909613488351

Guardiola, J., Gonzalez-Gomez, F., Garcia-Rubio, M. A., \& Lendechy-Grajales, A. (2012). Does higher income equal higher levels of happiness in every society? The case of the Mayan people. International Journal of Social Welfare, 22(1), 35-44. doi:10.1111/j.1468-2397.2011.00857.x

International Wellbeing Group. (2006). Personal wellbeing index-Adult. Melbourne, Vic: Australian Centre on Quality of Life. Recuperado de http://www.acqol.com.au/iwbg/wellbeing-index/pwi-a-english.pdf

Hochman, O., \& Skopek, N. (2013). The impact of wealth on subjective wellbeing: A comparison of three welfare-state regimes. Research in Social Stratification and Mobility, 34, 127-141. doi:10.1016/j.rssm.2013.07.003

Howell, R. T., \& Howell, C. J. (2008). The relation of economic status to subjective well-being in developing countries: A meta-analysis. Psychological Bulletin, 134(4), 536-560. doi:10.1037/0033-2909.134.4.536

Huebner, E. S. (2004). Research on assessment of life satisfaction of children and adolescents. Social Indicators Research, 66, 3-33. doi:10.1023/B:SOCI.0000007497.57754.e3

Kerstenetsky, C. L. (2000). Desigualdade e pobreza: Lições de Sen. Revista Brasileira de Ciências Sociais, 15(42), 113-122.

Lelli, S. (2001). Factor analysis vs. Fuzzy sets theory: Assessing the influence of different techniques on sen's functioning approach. Recuperado de http://econpapers.repec.org/paper/wpepapers/ces0121.htm

Luttmer, E. (2005). Neighbors as negatives: Relative earnings and well-being. Quarterly Journal of Economics, 120, 963-1002. doi: $10.1162 / 003355305774268255$

Machado, W. L., \& Bandeira, D. R. (2012). Bem-estar psicológico: definição, avaliação e conceitos correlatos. Estudos de Psicologia, 29(4), 587-595. 
Margolis, R., \& Myrskylae, M. (2013). Family, money, and health: Regional differences in the determinants of life satisfaction over the life course. Advances in Life Course Research, 18(1), 115-126. doi: $10.1016 /$ j.alcr.2013.01.001

Martos, T., \& Kopp, M. (2012). Life goals and well-being: Does financial status matter? Evidence from a representative hungarian sample. Social Indicators Research, 105, 561-568. doi:10.1007/s11205-011-9788-7

Montero, M. (2006). Hacer para transformar: El método en Psicología Comunitaria. Paidós: Buenos Aires.

Montserrat, C., Casas, F. A., \& Moura Jr, J. F. (2015). Children's subjective wellbeing in disadvantaged situations. In E. Fernandez, A. Zeira, T. Vecchiato, \& C. Canali (Eds.), Theoretical and empirical insights into child and family poverty, children's well-being: Indicators and research 10, (pp. 111-126). New York, NY: Springer.

Moura Jr., J. F., Ximenes, V. M., \& Sarriera, J. C. (2014). A construção opressora da pobreza no Brasil e suas consequências no psiquismo. Quaderns de Psicologia, 16(2), 85-93. doi:10.5565/rev/qpsicologia.1174

Oxford Poverty and Human Development Initiative. (2016). Global Multidimensional Poverty Index Databank. OPHI, University of Oxford

Paloutzian, R., \& Ellison, C. W. (1982). Loneliness, spiritual well-being, and quality of life. In L. Peplau, \& D. Perlman (Eds.), Loneliness: A Sourcebook of Current Theory, Research and Therapy (pp. 204-233). New York, NY: Wiley Interscience.

Pedersen, P.V., Grønbæk, M., \& Curtis, T. (2012). Associations between deprived life circumstances, wellbeing and self-rated health in a socially marginalized population. European Journal of Public Health, 22(5), 647-652. doi:10.1093/eurpub/ckr128

Perry, B. L., Pullen, E. L., \& Oser, C. B. (2012). Too Much of a Good Thing? Psychosocial resources, gendered racism, and suicidal ideation among low socioeconomic status African American women. Social Psychology Quarterly, 75(4), 334-359. doi: 10.1177/0190272512455932

Pitt, R., Sherman, J., Macdonald, M. A. (2016). Low-income working immigrant families in Quebec: Exploring their challenges to well-being, 106(8), 539545. doi:10.17269/CJPH.106.5028

Prady, S. L., Bloor, K., Bradshaw, J., Tunstall, H., Petherick, E. S., \& Pickett, K. (2015). Does administrative data reflect individual experience? Comparing an index of poverty with individually collected data on financial well-being in a multi-ethnic community. Social Policy and Society, 15(4), 513-535. doi: $10.1017 /$ S1474746415000597

Ryan, R. M., \& Deci, E. L. (2001). On Happiness and Human Potentials: A review of research on hedonic and eudaimonic well-being. Annual Review of Psychology, 52, 141-166. doi:10.1146/annurev.psych.52.1.141 
Rojas, M. (2011). Poverty and psychological distress in Latin America. Journal of Economic Psychology, 32, 206-217. doi:10.1016/j.joep.2010.01.014

Samman, E. (2007). Psychological and subjective wellbeing: A proposal for internationally comparable indicators. Oxford Poverty \& Human Development Initiative, OPHI. Recuperado de http://www.ophi.org.uk/wpcontent/uploads/OPHI-wp05.pdf

Salama, P., \& Destremau, B. (2002). Medidas de pobreza desmedida: Economía política de la distribución del ingreso. Santiago, Stg: LOM Ediciones.

Sarriera, J. C. (2015). O bem-estar sócio-comunitário: Bases conceituais e de pesquisa. In J. C. Sarriera, E. T. Saforcada, \& J. I. Alfaro (Eds.), Perspectiva psicossocial na saúde comunitária: A comunidade como protagonista (pp. 63-88). Porto Alegre, RS: Sulina.

Sen, A. K. (1996). Capacidad y bienestar. In M. C. Nussbaum, \& A. K. Sen (Eds.), La calidad de vida (pp. 23-45). México: FCE.

Sen, A. K. (2000). Desenvolvimento como liberdade. São Paulo, SP: Companhia das Letras.

Sen, A. (2013). What's happening in Bangladesh? The Lancet, 382, 1966-1968. doi:10.1016/S0140-6736(13)62162-5

Smith, L., \& Romero, S. (2010). Psychological interventions in the context of poverty: Participatory action research as practice. American Journal of Orthopsychiatry, 80(1), 12-25. doi:10.1111/j.1939-0025.2010.01003.x

Soares, S. S. D. (2009). Metodologias para estabelecer a linha de pobreza: Objetivas, subjetivas, relativas, multidimensional. Rio de Janeiro, RJ: IPEA.

Spaan, M. (2007). Evolution of a test item. Language Assessment Quarterly, $4(3), 279-293$.

Teschl, M., \& Comim, F. (2005). Adaptive preferences and capabilities: Some preliminary conceptual explorations. Review of Social Economy, 63(2), 229247. doi:10.1080/00346760500130374

Tiliouine, H., Cummins, R. A., \& Davern, M. (2006). Measuring wellbeing in developing countries: The case of Algeria. Social Indicators Research, 75, 1-30. doi: 10.1007/s11205-004-2012-2

Tomyn, A. J., Tyszkiewicz, M. D. F., \& Cummins, R. A. (2013). The personal wellbeing index: Psychometric equivalence for adults and school children. Social Indicators Research, 110, 913-924. doi:10.1007/s11205-011-9964-9

Wang, X., Shang, X., \& Xu, L. (2011). Subjective well-being poverty of the elderly population in China. Social Policy \& Administration, 45(6), 714-731. doi:10.1111/j.1467-9515.2011.00804.x

Watson, D., Clark, L., \& Tellegen, A. (1988). Development and validations of brief measures of positive and negative affect: The PANAS scales. Journal of Personality and Social Psychology, 54(6), 1063. doi:10.1037/00223514.54.6.1063 
Wilkinson, R. G., \& Pickett, K. E. (2006). Income inequality and population health: A review and explanation of the evidence. Social Science and Medicine, 62, 1768-1784. doi:10.1016/j.socscimed.2005.08.036

Webb, D. (2009). Subjective wellbeing on the Tibetan Plateau: An exploratory investigation. Journal of Happiness Studies, 10(6), 753-768. doi:10.1007/s10902-008-9120-7

Zoltowski, A. P. C., Costa, A. B., Teixeira, M. A. P., \& Koller, S. H. (2014). Qualidade metodológica das revisões sistemáticas em periódicos de psicologia brasileiros. Psicologia: Teoria e Pesquisa, 30(1), 97-104. doi:10.1590/1413-81232015208.10762014

Sobre os autores

James Ferreira Moura Junior é Doutor em Psicologia pela Universidade Federal do Rio Grande do Sul, Professor da Universidade da Integração Internacional da Lusofonia Afro-Brasileira (UNILAB) e do Programa de Pós-Graduação em Psicologia da Universidade Federal do Ceará (UFC). Coordenada a Rede de Estudos e Afrontamentos das Pobrezas, Discriminações e Resistências (reaPODERE).james.mourajr@unilab.edu.br Jorge Castellá Sarriera é Doutor em Psicologia Social pela Universidade Autonoma de Madrid e Professor do Programa de Pós-Graduação em Psicologia da Universidade Federal do Rio Grande do Sul. Coordena o Grupo de Pesquisa em Psicologia Comunitária (GPPC). jorgesarrieral@gmail.com

Recebido em: 28/07/2016

Revisado em: 16/01/2017

Aceito em: $12 / 02 / 2017$ 